\title{
Judgment Criterion of Cherenkov Radiation by an Accelerated Electron Bunch in Poloidal Magnetized Plasma
}

\author{
Wei Tian ${ }^{1,2, *}$, Shi-xiu Chen ${ }^{1}$, Ji-xiong Xiao ${ }^{1}$, Fei Gai ${ }^{1}$ and Kun Chen ${ }^{1}$ \\ ${ }^{1}$ School of Electrical Engineering, Wuhan University, Wuhan 430072, Hubei, China \\ ${ }^{2}$ School of Computer Science, South-Central University for Nationalities, Wuhan 430074, Hubei, China
}

Received: 30 Jun. 2013, Revised: 4 Nov. 2013, Accepted: 5 Nov. 2013

Published online: 1 May. 2014

\begin{abstract}
In order to make a great contribution for the breakthrough and fill gaps in field of radiation by electron bunch in poloidal magnetized plasma, judgment criterion of Cherenkov radiation by an accelerated electron bunch moving in Hermitian medium of poloidal magnetized plasma are presented in the paper. Through establishment of experimental model of Hermitian medium, radiation mechanism is analyzed. Eigenvalues are obtained. Through analysis of eigenvalues, dispersion relation is derived. Meanwhile, generation condition of Cherenkov radiation is summarized. It is found that plasma frequency affect the eigenvalue. However, only when cyclotron frequency is large enough, it can also affect the eigenvalue greatly. Finally, under the premise of fulfillment of judgment criterion of Cherenkov radiation, frequency dependence of Cherenkov radiation angle is achieved by computer calculation. The theoretical analysis and calculation will provide for further research of the new type UWB microwave radiator.
\end{abstract}

Keywords: Cherenkov radiation, poloidal magnetized plasma, eigenvalue, condition of radiation

\section{Introduction}

In recent years, our lab has researched and developed a new type UWB microwave radiator-pulsed plasma ultra-wide-band (UWB) microwave radiator [1]. The radiator has characteristics: ultra wide band, angles between the direction of maximum value and $\mathrm{z}$ axis change with the frequency of radiation wave, and so on. These characteristics are different from those of the general UWB microwave source. The radiation originates from Cherenkov radiation of an electron bunch moving in poloidal magnetized plasma.

The electron bunch moves with acceleration in the radiator. However, in view of references [2]-[9] only researching on Cherenkov radiation by an electron bunch moving with uniformly at certain speed, we had only researched on Cherenkov radiation by an electron bunch with uniform motion in poloidal magnetized plasma in order to simplify analysis. So, in this paper, Cherenkov radiation by an accelerated electron bunch moving in Hermitian medium of poloidal magnetized plasma is researched.
The paper is organized as follows. Section 1 is a brief introduction. In section 2 the experimental model of Hermitian medium is established and radiation mechanism is investigated. In section 3 the condition of radiation is derived through analysis of eigenvalue. In section 4 and 5, dispersion relation and frequency dependence of Cherenkov angle are calculated and analyzed respectively. Finally, conclusion is given in section 6.

\section{Radiation mechanism}

Radiation equipment of pulsed plasma is composed of power supply, rectifying device, high-voltage pulsed capacitor $C_{p}$ of energy storage, trigger device and diode. Circuit diagram of experiment is shown in figure 1 . Structure diagram of triggered vacuum diode is shown in figure 2. The diode has three electrodes: anode, cathode and triggered electrode. Method of field-breakdown is adopted into breakdown between cathode and triggered electrode, then electrons of cathode plasma are

\footnotetext{
*Corresponding author e-mail: victor-tian888@163.com
} 


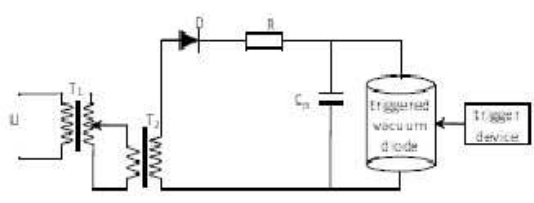

Fig. 1: Circuit diagram of experiment

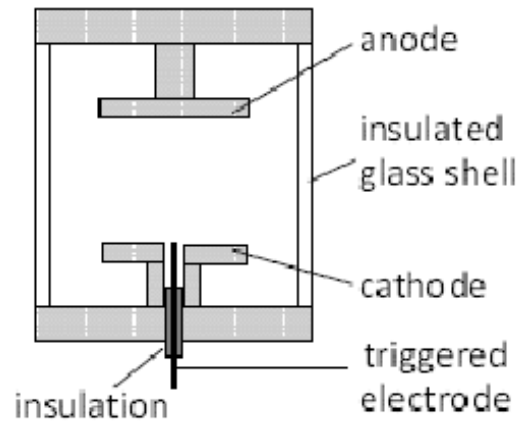

Fig. 2: Structure diagram of diode

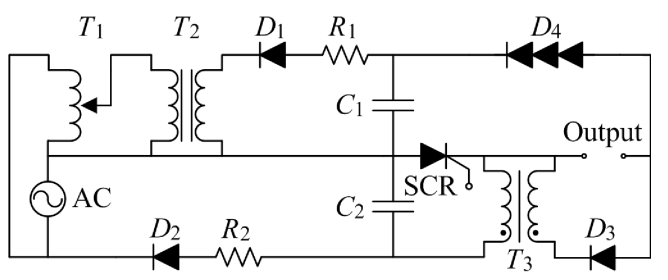

Fig. 3: Basic circuit of trigger current generator

accelerated by electric field of anode-cathode to form convection current[10]. The current excites poloidal magnetized field. Then the plasma is magnetized by the field to develop into Hermitian medium. In main circuit, two ends of $C_{p}$ connect anode and cathode of diode respectively. Two ends of trigger device connect triggered electrode and cathode respectively.

The circuit of trigger device, shown in figure 3, consists of current sustaining loop and pulse forming loop. $D_{1}, D_{2}$ and $D_{3}$ are diodes. $R_{1}$ and $R_{2}$ are current-limiting resistance. $D_{4}$ is silicon stack with $60 \mathrm{kV}$ withstand voltage. SCR is a silicon-controlled rectifier as a switch. $C_{1}$ is freewheeling capacitor used for current sustains, which is capable of the generation of abundant initial plasmas during the breakdown of trigger electrode, and $C_{2}$ is the capacitor used for pulse formation, respectively.

In the radiation equipment, electrons are not absolutely uniformly accelerated. However, in order to simplify analysis, it is supposed that electrons move with uniform acceleration along $\mathrm{z}$ axis in cylindrical coordinate. At the same time, the following analysis is based on the assumption that $u_{0}$ (initial velocity) $<<u$ (velocity after accelerated), namely acceleration is large enough.

The dielectric tensor of poloidal magnetized plasma with uniformly accelerated electrons is as follows [1]:

$$
\varepsilon=\left[\begin{array}{ccc}
\varepsilon_{1} & 0 & j \varepsilon_{2} \\
0 & \varepsilon_{3} & 0 \\
-j \varepsilon_{2} & 0 & \varepsilon_{1}
\end{array}\right]
$$

where $\varepsilon_{1}=\varepsilon_{0}\left(1+\frac{\omega_{p e}^{2}}{\omega_{c e}^{2}-2.25 \omega^{2}}\right), \varepsilon_{2}=\varepsilon_{0} \frac{\omega_{p e}^{2} \omega_{c e}}{1.5 \omega\left(\omega_{c e}^{2}-2.25 \omega^{2}\right)}$

$$
\omega_{c e} \equiv \frac{q_{e} B_{0}}{m_{e}} \omega_{p e}^{2} \equiv \frac{n_{e} q_{e}^{2}}{\varepsilon_{0} m_{e}}, \varepsilon_{3}=\varepsilon_{0}\left(1-\frac{\omega_{p e}^{2}}{2.25 \omega^{2}}\right),
$$

where $\omega_{p e}$ is plasma electron frequency of background plasma, $\omega_{c e}$ is electron cyclotron frequency. Collision effect is neglected. Beam-wave interaction in magnetized plasma is described by Maxwell's equations:

$$
\begin{gathered}
\nabla \times \vec{E}=-j \omega \mu_{0} \vec{H} \\
\nabla \times \vec{H}=j \omega \varepsilon_{0} \vec{D}+\vec{J} \\
\nabla \bullet \vec{D}=\rho \\
\nabla \bullet \vec{B}=0
\end{gathered}
$$

where the wave factor is $\exp \left\{j\left(\omega t-k_{z} z-m \varphi\right)\right\}$.

In the absence of the driving beam, the equations can be written as [11]:

$$
\nabla_{\perp}^{2} B_{z}+a_{1} B_{z}=a_{2} E_{z}
$$

$$
\nabla_{\perp}^{2} E_{z}+a_{3} E_{z}=a_{4} B_{z}
$$

where $\nabla_{\perp} \equiv \nabla-\vec{e}_{z} \partial / \partial z$,

$$
\left.\begin{array}{l}
a_{1}=-\frac{m^{2}\left(0.25 k_{z}^{2}-\kappa_{3}\right)}{r^{2}\left(0.25 k_{z}^{2}-\kappa_{1}\right)}-\left(0.25 k_{z}^{2}-\kappa_{3}\right) \\
a_{2}=j \frac{m}{r} \frac{0.5 k_{z}\left(\kappa_{1}-\kappa_{3}\right)}{\omega\left(0.25 k_{z}^{2}-\kappa_{1}\right)} \frac{\partial}{\partial r}+\frac{m}{r} \frac{\kappa_{2}\left(0.25 k_{z}^{2}-\kappa_{3}\right)}{\omega\left(0.25 k_{z}^{2}-\kappa_{1}\right)} \\
a_{3}=-\frac{m^{2}}{r^{2}} \frac{\kappa_{3}\left(0.25 k_{z}^{2}-\kappa_{1}\right)}{\kappa_{1}\left(0.25 k_{z}^{2}-\kappa_{3}\right)}+\frac{k_{2}^{2}}{\kappa_{1}}-0.25 k_{z}^{2}+\kappa_{1} \\
a_{4}=j \frac{m}{r} \frac{\omega 0.5 k_{z}\left(\kappa_{1}-\kappa_{3}\right)}{\kappa_{1}\left(0.25 k_{z}^{2}-\kappa_{3}\right)} \frac{\partial}{\partial r}-\frac{m}{r} \frac{\omega \kappa_{2}}{\kappa_{1}}
\end{array}\right\}
$$

$$
\kappa_{1}=2.25 \omega^{2} \mu_{0} \varepsilon_{1}, \kappa_{2}=2.25 j \omega^{2} \mu_{0} \varepsilon_{2}, \kappa_{3}=2.25 \omega^{2} \mu_{0} \varepsilon_{3}
$$

Equations (3) and (4) are coupled. The equations can be cast into the following form:

$$
\left(\nabla_{\perp}^{2}+p_{1}^{2}\right)\left(\nabla_{\perp}^{2}+p_{2}^{2}\right)\left(\begin{array}{c}
E_{Z} \\
B_{Z}
\end{array}\right)=0
$$

The value of $p_{1}$ and $p_{2}$ is respectively

$$
p_{1,2}^{2}=\frac{1}{2}\left\{\left(a_{1}+a_{3}\right) \pm\left[\left(a_{1}+a_{3}\right)^{2}-4\left(a_{1} a_{3}-a_{2} a_{4}\right)\right]^{\frac{1}{2}}\right\}
$$


It is shown that eigenvalues of $p_{1}$ and $p_{2}$ represent two modes respectively.

When the argument of $H_{0}^{(1)}\left(-p_{1,2} r\right)$ is very large, the radiation field can be expressed as approximate formulation:

$$
H_{0}^{(1)}\left(-p_{1,2} r\right) \approx \sqrt{2} \pi\left(-p_{1,2} r\right) e^{-j\left(p_{1,2} r-\pi / 4\right)}
$$

Only when $p$ is real, can the mode radiate. Or else the mode is a local mode. So it is obvious that, in order to make eigenvalues of $p$ real values, the following condition should be fulfilled:

$$
p_{1,2}^{2}>0
$$

\section{Analysis of eigenvalue}

In order to simplify equation (8), it is supposed that $m=0$. Equation (5) can be written as:

$$
\left.\begin{array}{l}
a_{1}=-\left(0.25 k_{z}^{2}-\kappa_{3}\right) \\
a_{2}=a_{4}=0 \\
a_{3}=\frac{\kappa_{2}^{2}}{\kappa_{1}}-\left(0.25 k_{z}^{2}-\kappa_{1}\right)
\end{array}\right\}
$$

Equation (9) can be now substituted into equation (7) with the result

$$
p_{1,2}^{2}=\frac{1}{2}\left\{\left(a_{1}+a_{3}\right) \pm \sqrt{\left(a_{1}-a_{3}\right)^{2}}\right\}
$$

(i) when $a_{1}>0$

In this condition, the following result can be received:

$$
p_{1}^{2}=a_{1}=2.25 \omega^{2} \mu_{0} \varepsilon_{0}\left(1-\omega_{p e}^{2} / 2.25 \omega^{2}\right)-0.25 k_{z}^{2}>0
$$

Equation (11) gives the following solution:

$$
\frac{u}{c}>\sqrt{\frac{1}{9-4 \omega_{p e}^{2} / \omega^{2}}}
$$

Because the equation of $\sqrt{\frac{1}{9-4 \omega_{p e}^{2} / \omega^{2}}}<1$ is easily satisfied, it can be concluded that, in some condition, $a_{1}$ could be bigger than 0 . So $p_{1}$ mode can exist and radiate, if the condition of equation (12) is satisfied.

(ii) when $a_{3}>0$

In this condition, if the following condition is fulfilled, another mode can radiate:

$$
\begin{aligned}
p_{2}^{2}= & a_{3}=-0.25 k_{z}^{2}-2.25 \frac{\omega^{2}}{c^{2}}\left[\frac{\omega_{c e}^{2} \omega_{p e}^{4}}{2.25 \omega^{2}\left(\omega_{c e}^{2}-2.25 \omega^{2}\right)\left(\omega_{c e}^{2}-2.25 \omega^{2}+\omega_{p e}^{2}\right)}\right. \\
& \left.-1-\frac{\omega_{p e}^{2}}{\omega_{c e}^{2}-2.25 \omega^{2}}\right]>0
\end{aligned}
$$

Equation (13) gives the following solution:

$$
\frac{u}{c}>\sqrt{\frac{1}{9+4 \frac{\omega_{p e}^{2}\left(2.25 \omega^{2}-\omega_{p e}^{2}\right)}{\omega^{2}\left(\omega_{c e}^{2}+\omega_{p e}^{2}-2.25 \omega^{2}\right)}}}
$$

It is same to condition (i), the condition (ii) is easily satisfied. So $p_{2}$ mode can exist and radiate, on this condition.

In general, it can be seen that two modes can radiate if equations of (12) and (14) hold. Or else, at least one mode is a local field moving with the charged particle and can not radiate. When only condition (i) is satisfied, there is only transverse component of electric field, namely there is only TE (Transverse Electric) wave. When only condition (ii) is satisfied, there is only transverse component of magnetic field, namely there is only TM(Transverse Magnetic) wave.

\section{Dispersion relation}

Equation (11) can be simplified:

$$
p_{1}^{2}=-0.25 k_{z}^{2}+2.25 \frac{\omega^{2}}{c^{2}}-\frac{\omega_{p e}^{2}}{c^{2}}>0
$$

So the dispersion relation of $p_{1}$ mode can be derived through above derivations:

$$
k_{1}^{2}=0.75 \frac{\omega^{2}}{u^{2}}+2.25 \frac{\omega^{2}}{c^{2}}-\frac{\omega_{p e}^{2}}{c^{2}}
$$

Then equation (13) can be also simplified:

$$
p_{2}^{2}=-0.25 k_{z}^{2}+2.25 \frac{\omega^{2}}{c^{2}}+\frac{\omega_{p e}^{2}\left(2.25 \omega^{2}-\omega_{p e}^{2}\right)}{c^{2}\left(\omega_{c e}^{2}-2.25 \omega^{2}+\omega_{p e}^{2}\right)}>0
$$

The dispersion relation can be also derived:

$$
k_{2}^{2}=0.75 \frac{\omega^{2}}{u^{2}}+2.25 \frac{\omega^{2}}{c^{2}}+\frac{\omega_{p e}^{2}\left(2.25 \omega^{2}-\omega_{p e}^{2}\right)}{c^{2}\left(\omega_{c e}^{2}-2.25 \omega^{2}+\omega_{p e}^{2}\right)}
$$

By comparison of equation (16) and (18), if $\omega_{c e}<<$ $\omega_{p e}$, equation (18) can be simplified into equation (16).

\section{Calculation of Cherenkov angle}

Based on equation (17), the Cherenkov angle $\theta_{c}$ can be written as:

$\theta_{c}=\arctan \left\{\frac{u^{2}}{c^{2}}\left[2.25+\frac{\omega_{p e}^{2}\left(2.25 \omega^{2}-\omega_{p e}^{2}\right)}{\omega^{2}\left(\omega_{c e}^{2}+\omega_{p e}^{2}-2.25 \omega^{2}\right)}\right]-\frac{1}{4}\right\}^{\frac{1}{2}}$

Above equation is normalized as:

$$
\theta_{c}=\arctan \left\{\left[\bar{u}^{2}\left(2.25+\frac{2.25 \bar{\omega}^{2}-1}{\bar{\omega}^{2}\left(\bar{\omega}_{c e}^{2}+1-2.25 \bar{\omega}^{2}\right)}\right)\right]-\frac{1}{4}\right\}^{\frac{1}{2}}
$$

Where $\bar{\omega}_{c e}=\omega_{c e} / \omega_{p e} ; \bar{u}_{0}=u_{0} / c \bar{\omega}=\omega / \omega_{p e}$;

Here, Cherenkov radiation diagram is shown in figure 4. 


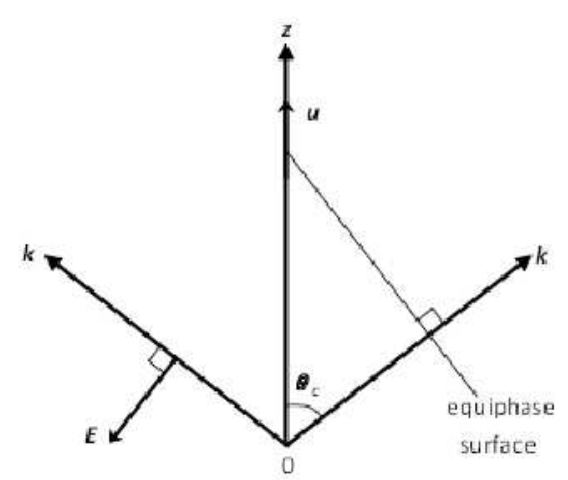

Fig. 4: Cherenkov radiation

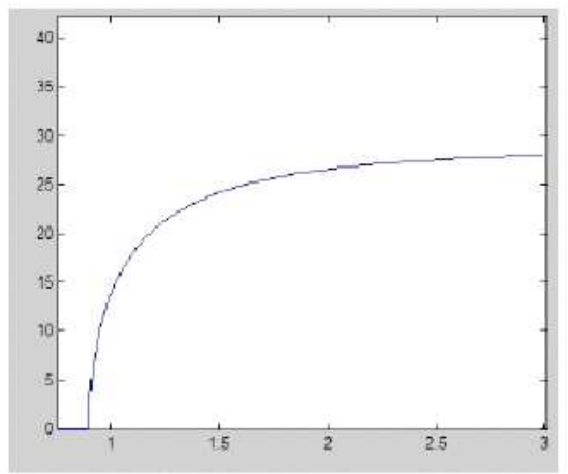

Fig. 5: Frequency dependence of Cherenkov angle for $\omega_{p e}=$ $0.29 \times 10^{1} 1, \omega_{c e} / \omega_{p e}=0.1, u / c=0.5$

Combined with figure 3, equation (20) shows that Cherenkov angle increases with increase of $u_{0}$, which is consistent to Cherenkov theory in reference[12] though there are different mediums.

At the same time, figures $5 \sim 8$ show frequency dependence of Cherenkov angle in different conditions.

From figures 4 and 5, it can be observed that frequency dependence of Cherenkov angle is almost unchanged with increase of $\bar{\omega}_{c e}$ to 0.155 , because of $\omega_{c e}<<\omega_{p e}$.

By comparison of figures 5, 7 and 8 , it can be seen that distribution of Cherenkov angle is expanded, and frequency band of radiation $\left(0^{\circ}<\theta_{c}<90^{\circ}\right)$ is wider with increase of $u$.

In addition, from above figures, it can be observed that Cherenkov angle will research a certain value with increase of $\omega$.

\section{Conclusion}

Theoretical research and numerical calculation on Cherenkov radiation of an accelerated electric bunch in

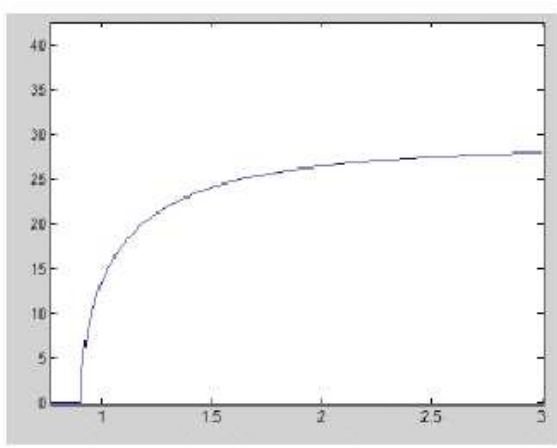

Fig. 6: Frequency dependence of Cherenkov angle for $\omega_{p e}=$ $0.29 \times 10^{1} 1, \omega_{c e} / \omega_{p e}=0.155, u / c=0.5$

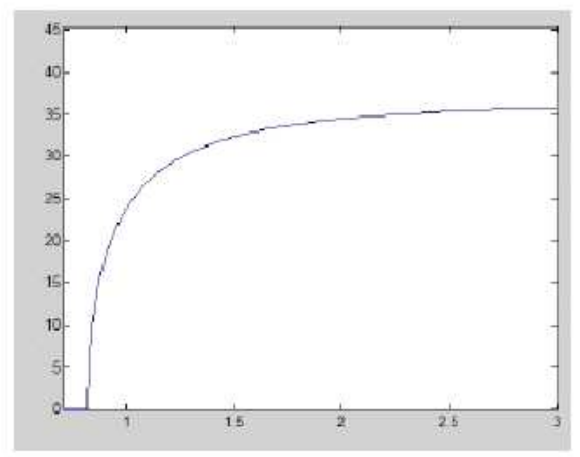

Fig. 7: Frequency dependence of Cherenkov angle for $\omega_{p e}=$ $0.29 \times 10^{1} 1, \omega_{c e} / \omega_{p e}=0.1, u / c=0.55$

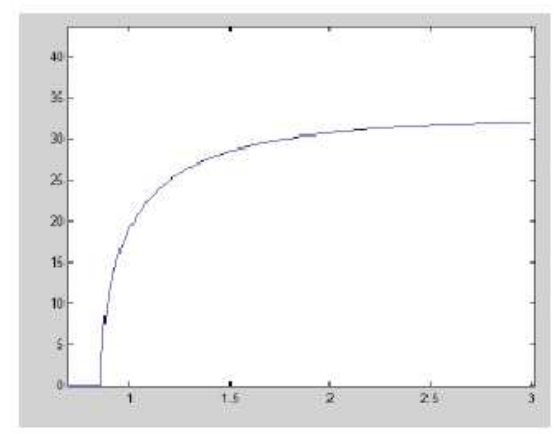

Fig. 8: Frequency dependence of Cherenkov angle for $\omega_{p e}=$ $0.29 \times 10^{1} 1, \omega_{c e} / \omega_{p e}=0.1, u / c=0.6$

poloidal magnetized plasma are presented in this paper. The following results can be received:

(1) In the Hermitian medium, both of two modes can radiate on the condition of $p_{1,2}^{2}>0$. Or else, there is only TM wave or TE wave.

(2) Generation condition of Cherenkov radiation is derived. The condition is $p_{1}^{2}$ or $p_{2}^{2}>0$. 
(3) Through the analysis of dispersion relation and frequency dependence of Cherenkov angle, when value of $u$ increases, Cherenkov angle will be expanded.

\section{Acknowledgments}

The authors would like to thank the National Natural Science Foundation of China under Grant No. 51207171 and No.11075123 for financially supporting this research.

\section{References}

[1] Chen Shi-xiu, Sun You-lin, Xia Chang-zheng, Yan Guozhi, Preliminary study on mechanism of radiating microwave from pulsed plasma, High Power Laser and Particle Beams, 20, 477-481 (2008).

[2] P. A. Cherenkov, Dokl. Akad Nauk SSSR (RUSSIA), 2, 451 (1934)

[3] GGFazio, JVJelley, WNCharman, Genration of Cherenkov light flashes by cosmic radiation within eyes of Apollo astronauts, Nature, 228, 260 (1970).

[4] Liu Shenggang Zhang Ping Liu Weihao, et al, Surface polariton Cherenkov light radiation source Physical Review Letters, 109, 153902 (2012).

[5] K. Yasumoto, H.Shigematsu Analysis of propagation characteristics of radio waves in tunnels using a surface impedance approximation, Radio Science, 19, 597-602 (1984).

[6] J. Zheng C. X. Yu Cherenkov radiation generated by a beam of electrons revisited Physics of Plasmas 12, 093105 (2005)

[7] Limin Li, Gouxin Cheng, Le Zhang, et al, Role of the rise rate of beam current in the microwave radiation of vircator, Journal of Applied Physics, 109, 074504 (2011).

[8] LIU Sheng-gang, ZHANG Ya-xin, YAN Yang, et al Cherenkov radiation by an electron bunch moving in Hermitian medium Journal of Applied Physics, 102, 044901 (2007).

[9] ZHANG Ya-xin, JIA Jia, and FU Qiang Simulation of Cherenkov Radiation in Magnetized Plasma by a Charged Particle Journal of University of Electronic Science and Technology of China, 38, 549-552 (2009).

[10] Gai Fei, Chen Shixiu, Chen Kun, Li Jun, Tian Wei, Xiao Jixiong, Conduction characteristics of long-gap triggered vacuum switch, High power laser and particle beams, 24, 847-850 (2012).

[11] D. G. Swanson Plasma Waves Academic Press, New York, (2003).

[12] Jin Au Kong Electromagnetic Wave Theory, EMW Publishing, USA, (2008).

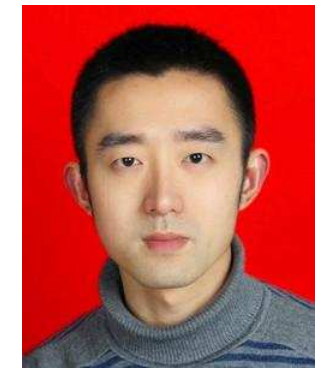

Wei Tian was born in Hubei, China, in 1979. He received the B.S. degree from Hubei University of Technology, Wuhan, China, in 2002 and M.S. degree from Wuhan University of Technology, Wuhan, China, in 2006. He works at South-Central University for Nationalities. He is currently working toward the Ph. D. in Wuhan University, Wuhan, China. He is engaged in the study of high power microwave and discharge plasma physics.

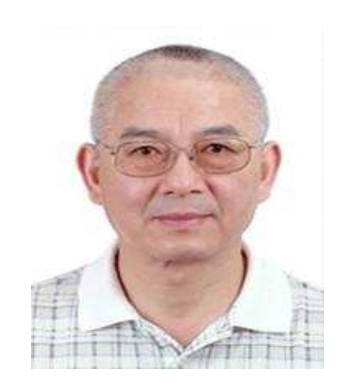

Shixiu Chen was born in Hubei, China, in 1951. He received the $\mathrm{Ph}$. D. degree from Wuhan University, Wuhan, China, in 1999. $\mathrm{He}$ is currently a Professor at Wuhan University, Wuhan, China. He is engaged in the study of high voltage engineering and discharge

plasma physics.

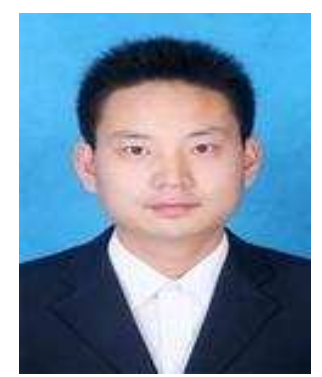

Jixiong Xiao was born in Hunan, China, in 1981. He received the B.S. degree from Hunan Institute of Science and Technology, Yueyang, China, in 2005 and M.S. degree from Wuhan University of Technology, Wuhan, China, in 2009. He is currently working toward the $\mathrm{Ph}$. D. in Wuhan University, Wuhan, China. $\mathrm{He}$ is engaged in the study of high power microwave.

Fei Gai was born

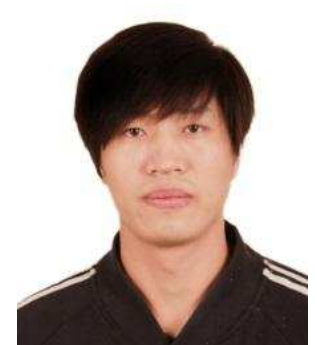
in Shandong, China, in 1983. He received the B.S. and M.S. degree from University of Jinan, Jinan, China, in 2005 and 2009, respectively. $\mathrm{He}$ is currently working toward the $\mathrm{Ph}$. D. in Wuhan University, Wuhan, China. He is engaged in the study of high voltage engineering and pulsed power technology. 